\title{
HPV vaccines for prevention of cervical pre-cancer in adolescent girls and women
}

\begin{abstract}
Jia Jian Li' ${ }^{1}$ Jessica Stetz ${ }^{2}$
${ }^{1}$ Corresponding author. Downstate Medical Center, State University of New York. New York City, New York, United States of America. ORCID: 0000-0002-4446-9412. jiajian.li@downstate.edu ${ }^{2}$ Downstate Medical Center, State University of New York. New York City, New York, United States of America. ORCID: 0000-0001-6183-2925. stetzjessica@yahoo.com
\end{abstract}

\begin{tabular}{|l|l|}
\hline The NNT Color recommendation & Green (benefits > harms) \\
\hline Summary Heading & $\begin{array}{l}\text { Significantly reduces the risk of cervical pre-cancer in women } 15 \text { to } 25 \\
\text { years old }\end{array}$ \\
\hline Benefits in NNT & $\begin{array}{l}\text { - NNT of } 60 \text { for preventing one cervical pre-cancer (women } 15 \text { to } 25 \\
\text { years old with or without HPV infection) }\end{array}$ \\
\hline Benefits in Percentages & $\begin{array}{l}-1.7 \% \text { lower risk of cervical pre-cancer (women } 15 \text { to } 25 \text { years old with } \\
\text { or without HPV infection) }\end{array}$ \\
\hline Harms in NNT (NNH) & $\begin{array}{l}\text { Not applicable: No significant difference between the vaccinated and } \\
\text { unvaccinated individuals }\end{array}$ \\
\hline Harms in Percentages & $\begin{array}{l}\text { Not applicable: No significant difference between the vaccinated and } \\
\text { unvaccinated individuals }\end{array}$ \\
\hline Efficacy Endpoints & Pre-cancer cervical lesions, cervical cancer \\
\hline Harm Endpoints & $\begin{array}{l}\text { Serious adverse events, death, pregnancy-related adverse events } \\
\text { (spontaneous abortion, stillbirth, congenital malformations) }\end{array}$ \\
\hline
\end{tabular}




\section{Narrative}

Human papillomaviruses (HPV) are sexually transmitted infections that are common in young people. In the United States, HPV infects nearly 80 million people. Approximately, one in four Americans are currently infected with HPV. About 14 million individuals become infected with HPV each year in the United States ${ }^{1}$. Usually these viruses are cleared by the immune system and pose little threat. However, highrisk (hr) types of HPV can cause persistent infection which can lead to precancerous changes in cells. Precancerous changes are defined as infection of at least two thirds of the surface layer of cervical cells. Some people with this pre-cancerous abnormality will go on to develop cervical cancer; unfortunately, we don't know which individuals with infection will clear these changes and which will develop cancer. A number of different hrHPV types cause cervical pre-cancer and cancer, the most important of which are HPV16 and 18. These high-risk types cause about $70 \%$ of cervical cancers worldwide ${ }^{2}$. Cervical cancer is the fourth most common cause of cancer in women worldwide, and $86 \%$ of worldwide cervical cancer cases occur in developing countries. The lifetime risk of cervical cancer is approximately $0.6 \%$ and the chance of dying from cervical cancer is approximately $0.2 \%^{3}$. Vaccination with HPV-like particles triggers the production of antibodies which protect against the development of precancerous cervical changes. The Cochrane review discussed here assesses the benefits and harms of prophylactic HPV vaccination against cervical pre-cancer and HPV16/18 infections in adolescent girls and women ${ }^{2}$. The assumption is that if HPV vaccine effectively prevents precancerous changes by hrHPV infection, vaccination should prevent a majority of cases of cervical cancer.

A total of 26 studies involving 73,428 adolescent girls and women were included in the Cochrane metaanalysis. The authors performed pre-planned analyses separately in groups of women $15-25$ and $26-45$, as the majority of trials enrolled subjects 25 years of age or younger ${ }^{2}$. Participants were divided into sub-groups; the first group had no evidence of baseline infection with high-risk human papillomaviruses types, the second group had no evidence of baseline infection with HPV types included in the vaccines, and the third group had unknown baseline infection with HPV. The review included all trials that evaluated vaccine safety over a period 0.5 to 7 years and ten trials with follow-up 3.5 to 8 years. Cervical cancer could not be evaluated as an outcome due to inadequate study sample sizes and insufficient follow up duration ${ }^{2}$. The summary of the Cochrane review analyses results is presented in the table.

There were no significant differences in serious adverse events between vaccinated and unvaccinated individuals (RR: $0.98,95 \% \mathrm{Cl} 0.92$ to $1.05 ; 71,597$ participants; high-quality evidence) independent of type or dosage of HPV vaccine. The HPV vaccine administration was not associated with increased risk of pregnancy-related adverse outcomes either (moderate to high quality evidence).

\section{Caveats}

Vaccination against HPV is performed to reduce the incidence of cervical cancer. Unfortunately, this outcome could not be assessed in the meta-analysis, because the included trials lacked sufficient power and adequate follow up ${ }^{2}$. Additionally, this metaanalysis extracted efficacy data from peer-reviewed published reports. Incomplete reporting of the original trials limited the number of studies that could be included in each of the analyses ${ }^{2}$. Furthermore, HPV vaccines were not yet standardized at the time of the studies so the potency for specific anti-HPV serological responses may be a variable ${ }^{4}$.

The review identified several notable biases. Computed efficacy estimates for women who received only one or two doses were calculated instead of measured. Another post hoc analysis limitation was the variation caused by counting events for participants who received at least one dose at day one, compared to counting events for participants who received all three doses on the day of the last administration ${ }^{2}$. Another source of bias originated from the fact that for several outcomes no information was available for mid-adult women. Finally, the comparison of the risks of adverse events was compromised by the use of different products administered to participants in the control group, varying from adjuvant (often aluminum hydroxide or other aluminum compound) or an alternative vaccine (often Hepatitis $A$ or Hepatitis $B)^{5}$, and therefore, the pooled risks of adverse effects associated with HPV vaccines and the assumed risks for control groups must be interpreted carefully. It must be noted that 25 out of the 26 trials were 
sponsored by the vaccine manufacturers. However, the analyses of bias performed by the Cochrane review found little evidence of important bias due to trial sponsorship.

The quality of the evidence was deemed to be high, since the data included more than 70,000 women from randomized trials ${ }^{2}$. Heterogeneity was minimal in this review since data series did not combine participants with different baseline HPV status (presence of hrHPV DNA, presence of DNA of the HPV vaccine types). Moreover, younger (15 to 26 years) women were distinguished from mid-adult women ( 24 to 45 years). Efficacy estimates were not significantly different by vaccine type, and jointly pooled estimates were retained. Only when significant heterogeneity by vaccine types was noted, were separate efficacy estimates by vaccine type pooled ${ }^{2}$. When significant heterogeneity persisted, the review used meta-regression to investigate sources of heterogeneity, such as serological status, study design items, study size and sexual history ${ }^{6,7}$. This process decreased any heterogeneity presented in the data.

While the reason for higher efficacy of HPV vaccine in younger women is not discussed in the Cochrane review, it is likely that younger age allows for more likelihood of exposure to HPV and potentially developing pre-cancerous cervical lesions. This factor could justify the recommendations that HPV vaccines should be administered as early as possible in adolescence. Finally, it is important to remember that the lifetime risk of cervical cancer is approximately $0.6 \%^{3}$. So even if the vaccine is $100 \%$ effective at preventing cervical cancer, the best NNT for cancer prevention over a lifetime would be 167.

As of December 2018, the cost of HPV vaccine is approximately $\$ 261-\$ 461$.

\section{Conclusion}

The evidence presented in this meta-analysis shows the HPV vaccination confers significant benefit in preventing cervical pre-cancer. The effect is higher for lesions associated with HPV16/18. The data also demonstrates an absence of serious adverse events. Therefore, we have assigned a color recommendation of Green (Benefit > Harm) to this vaccine.

Table. Efficacy of HPV vaccine for preventing cervical pre-cancer in different subgroups based on age and type of cervical abnormality

\begin{tabular}{|c|c|c|}
\hline Subgroups of women aged $\mathbf{1 5 - 2 5}$ & Cervical pre-cancer* with HPV $16 / 18$ & Any cervical pre-cancer \\
\hline Women hrHPV negative & $\begin{array}{l}\text { ARD: } 1.6 \% \\
\text { NNT: } 63 \\
\text { RR: } 0.01 \text { (0 to } 0.05) \\
\text { Quality of Evidence: High }\end{array}$ & $\begin{array}{l}\text { ARD: } 1.8 \% \\
\text { NNT: } 56 \\
\text { RR: } 0.37(0.25 \text { to } 0.55) \\
\text { Quality of Evidence: High }\end{array}$ \\
\hline Women HPV $16 / 18$ negative & $\begin{array}{l}\text { ARD: } 1.07 \% \\
\text { NNT: } 94 \\
\text { RR: } 0.05(0.03 \text { to } 0.10) \\
\text { Quality of Evidence: High }\end{array}$ & $\begin{array}{l}\text { ARD: } 1.36 \% \\
\text { NNT: } 74 \\
\text { RR } 0.41 \text { ( } 0.32 \text { to } 0.52) \\
\text { Quality of Evidence: High }\end{array}$ \\
\hline $\begin{array}{l}\text { All Women with or without HPV } \\
\text { infection }\end{array}$ & $\begin{array}{l}\text { ARD: } 1.84 \% \\
\text { NNT: } 55 \\
\text { RR: } 0.46 \text { ( } 0.37 \text { to } 0.57) \\
\text { Quality of Evidence: High }\end{array}$ & $\begin{array}{l}\text { ARD: } 1.68 \% \\
\text { NNT: } 60 \\
\text { RR } 0.70 \text { ( } 0.58 \text { to } 0.85) \\
\text { Quality of Evidence: High }\end{array}$ \\
\hline \multicolumn{3}{|l|}{ Subgroups of women aged 25 to 45} \\
\hline Women free of hrHPV & No data & No data \\
\hline Women free of HPV $16 / 18$ & No data & No data \\
\hline $\begin{array}{l}\text { All Women with or without HPV } \\
\text { infection }\end{array}$ & $\begin{array}{l}\text { RR: } 0.74(0.52 \text { to } 1.05) \\
\text { Difference not significant } \\
\text { Quality of Evidence: moderate }\end{array}$ & $\begin{array}{l}\text { RR: } 1.04 \text { ( } 0.83 \text { to } 1.30) \\
\text { Difference not significant } \\
\text { Quality of Evidence: moderate }\end{array}$ \\
\hline
\end{tabular}

Abbreviations: hr: High risk; HPV: human papilloma virus; NNT: Number-needed-to-treat, ARD: Absolute risk difference; RR Relative risk

* Cervical intraepithelial neoplasia, grade 2 and higher (CIN2+). Data for CIN3+ are not reported. 


\section{Author contributions}

Li JJ and Stetz J designed the evidence-based review protocol. Li JJ drafted the manuscript. Stetz J reviewed the statistical analysis and edited the manuscript.

\section{Competing interests}

No financial, legal or political competing interests with third parties (government, commercial, private foundation, etc.) were disclosed for any aspect of the submitted work (including but not limited to grants, data monitoring board, study design, manuscript preparation, statistical analysis, etc.).

\section{References}

1. Centers for Disease Control and Prevention. Papilomavírus Humano (HPV). [Internet]. 2018. [accessed 12 nov. 2018]. Available at: https://www.cdc.gov/hpv/parents/vaccine.html.

2. Arbyn M, Xu L, Simoens C, Martin-Hirsch PP. Prophylactic vaccination against human papillomaviruses to prevent cervical cancer and its precursors. Cochrane Database Syst Rev. 2018;5(CD009069). doi: 10.1002/14651858.CD009069

3. American Cancer Society. Lifetime Risk of Developing or Dying From Cancer. [Internet]. 2018. [accessed 30 nov. 2018]. Available at: https://www.cancer.org/cancer/cancer-basics/lifetimeprobability-of-developing-or-dying-from-cancer.html

4. Unger E, Dillner J. Human Papillomavirus Laboratory Manual. 1st Edition. Geneva; 2010.

5. World Health Organization. Immunization Vaccines and Biologicals [Internet]. 2009. Available at: https://www.who.int/ immunization/research/en/

6. Thompson SG, Sharp SJ. Explaining heterogeneity in metaanalysis: a comparison of methods. Statistics in Medicine 1999;18:2693-708. doi: 10.1002/(SICI)1097-0258(19991030)

7. Sharp S. Meta-analysis regression. Statistics Technical Bulletin 1998;7(48):148-55.

8 GoodPx. [Internet]. 2018. [ accessed 1st dez. 2018]. Available at: https://www.goodrx.com/gardasil-9?drug-name=gardasil+9 